\title{
Magnetic Resonance Spectroscopy Study of Glycine Pathways in Nonketotic Hyperglycinemia
}

\author{
ANGĖLE VIOLA, BRIGITTE CHABROL, FRANÇOIS NICOLI, SYLVIANE CONFORT-GOUNY, \\ PATRICK VIOUT, AND PATRICK J. COZZONE \\ Center for Magnetic Resonance in Biology and Medicine CRMBM-UMR-CNRS 6612, Faculty of Medicine, \\ Marseille, France [A.V., F.N., S.C.-G., P.V., P.J.C.], and Neuropediatric Service, CHU la Timone, \\ Marseille, France [B.C.]
}

\begin{abstract}
ABST
Nonketotic hyperglycinemia is a life-threatening disorder
in neonates characterized by a deficiency of the glycine
cleavage system. We report on four cases of the neonatal form
of the disease, which were investigated by in vitro ${ }^{1} \mathrm{H}$ mag-
netic resonance spectroscopy of blood and cerebrospinal fluid,
and in vivo ${ }^{1} \mathrm{H}$ magnetic resonance spectroscopy of brain. The
existence of glycine disposal pathways leading to an increase
in lactate in fluids and creatine in fluids and brain was
demonstrated. This is the first observation of elevated creatine
in brain in nonketotic hyperglycinemia. A recurrent decrease
of glutamine and citrate was observed in cerebrospinal fluid,
which might be related to abnormal glutamine metabolism in
brain. Finally, the cerebral $N$-acetylaspartate to myo-inositol-
glycine ratio was identified as a prognostic indicator of the
disease. (Pediatr Res $\mathbf{5 2 :} \mathbf{2 9 2 - 3 0 0 , ~ 2 0 0 2 ) ~}$
\end{abstract}
NKH is an inborn error of autosomal recessive inheritance in which Gly catabolism shows a deficiency of the GCS causing the accumulation of Gly in all body tissues. The neonatal form of the disease is characterized by postnatal onset of lethargy, hypotonia, myoclonic jerks, and apnea generally leading to death during early infancy. The outcome of surviving patients is poor because they develop severe neurologic defects and mental retardation. The pathophysiology of NKH has been attributed to an overstimulation of the excitatory $\mathrm{N}$-methyl-D-aspartate receptors in the cerebral cortex $(1,2)$. NKH is characterized by elevated concentrations of Gly in plasma, CSF, and brain (3) with a $\mathrm{CSF}_{\text {glycine }}$ to

Received February 21, 2001; accepted November 8, 2001

Correspondence and reprint requests: Prof. P. J. Cozzone, CRMBM-UMR-CNRS 6612, Faculté de Médecine la Timone, 27, bvd Jean Moulin, 13005 Marseille, France; e-mail: patrick.cozzone@medecine.univ-mrs.fr

Supported by grants from CNRS (Centre National de la Recherche Scientifique) and ADEREM (Association pour le Développement des Recherches Biologiques et Médicales au CHR de Marseille).

DOI: 10.1023/01.PDR.0000019549.97278.EF
Cho, choline
Abbreviations
tCr, total creatine
CSF, cerebrospinal fluid
DX, dextromethorphan
GA, gestational age
GCS, glycine cleavage system
Glx, glutamate + glutamine
Gly, glycine
GS, glutamine synthetase
Ins, myo-inositol
MRI, magnetic resonance imaging
MRS, magnetic resonance spectroscopy
NAA, $N$-acetylaspartate
NKH, nonketotic hyperglycinemia
SB, sodium benzoate

plasma $_{\text {glycine }}$ ratio greater than 0.08 . The diagnosis is definitively established after measurement of hepatic GCS activity $(1,2)$. At autopsy, the brain GCS activity is considerably reduced or nil (2). This enzymatic defect has also been identified in fibroblasts (2) and lymphoblasts $(4,5)$, which suggests that NKH pathophysiology may result from a general metabolic problem. However, the emphasis in NKH case reports is indeed on brain damage. MRI of the brain generally shows dysgenesis of the corpus callosum, delay in myelination, vacuolation, and gliosis. Acute hydrocephalus has also been detected in rare cases (6). At autopsy, spongiform degeneration of white matter and astrocytic gliosis associated with neuronal loss are frequent $(7,8)$. None of the current treatments is satisfactory. Administration of SB/DX combination associated with Gly dietary restriction is one of the current therapies. SB conjugates Gly to form hippurate, which is excreted in urine, and DX is a moderate noncompetitive antagonist of the $N$-methyl-D-aspartate channel. However, the outcome of children responding to this therapy remains poor with severe retardation. 
Although the genetic basis of NKH disease has been extensively studied, all metabolic aspects of the NKH disorder have not been explored so far. Information on the impact of elevated Gly on brain metabolism and cerebral atrophy is still lacking. Apart from the increase in Gly, which was shown by in vivo MRS (9) or postmortem analysis of brain specimens, little is known about the cerebral metabolic consequences of the disease. In a recent paper, a depletion of cerebral D-serine in the cortex (10) was described. Moreover, an ${ }^{1} \mathrm{H}$ MRS study evidenced an increase in the resonance common to Glx in the frontal white matter (11). Taken together, these results suggest profound changes of brain metabolism in NKH disease. The aim of this study was to investigate metabolic disorders in NKH patients using high-resolution ${ }^{1} \mathrm{H}$ MRS of blood serum and CSF and localized in vivo ${ }^{1} \mathrm{H}$ MRS of the brain. This in vivo/in vitro concerted approach was designed to study metabolic alterations affecting the CNS whenever it was compatible with medical ethics and the constraints of the administration of a palliative treatment. We present a report on four cases of the neonatal form of NKH. The therapeutic course of the patients was routinely monitored by chromatography of amino acids. ${ }^{1} \mathrm{H}$ MRS of biologic fluids aided in elucidating original brain metabolite variations that amino acid chromatography could not fully explain. The correlation between in vitro and in vivo MRS observations led to the identification of biochemical perturbations, potentially involved in NKH pathophysiology, which had gone undetected in the past.

\section{METHODS}

Patients. A summary of the biochemical features of patients is reported in Table 1. All patients were born after uneventful pregnancy and delivery. All of them had hypotonia, apnea, myoclonic jerks, and seizures. EEG showed a burst suppression pattern in all cases. Patients were diagnosed with NKH on the basis of Gly concentration in blood and CSF and assessment of hepatic GCS activity (Table 1). Patient 1: This baby girl had general iminoglycinuria at birth [hydroxyproline, $335 \mathrm{mmol} / \mathrm{mol}$ creatine (normal, $<149 \mathrm{mmol} / \mathrm{mol}$ creatine) and proline, $82 \mathrm{mmol} / \mathrm{mol}$ creatine (normal $0-68 \mathrm{mmol} / \mathrm{mol}$ creatine)], probably because Gly, proline, and hydroxyproline share the same renal carrier. High levels of proline, hydroxyproline, serine, methionine, cysteine, and alanine were detected in blood. Alanine was elevated in CSF. Treatment with vigabatrin and phenobarbital reduced the frequency of seizures. At $10 \mathrm{mo}$, the administration of SB $(4 \mathrm{~g} / \mathrm{d})$ and DX $(40 \mathrm{mg} / \mathrm{kg})$ was instituted. Dosages were progressively increased to $6 \mathrm{~g} / \mathrm{d}$ of SB and $110 \mathrm{mg} / \mathrm{d}$ of DX. The patient has continued on this regimen to the present time and is currently (age 8 y) seizure free. Neurologic and psychomotor development improved slowly but significantly on treatment. She remains severely retarded, but she has a few milestones and shows signs of social behavior. Patient 2: This baby girl was unresponsive to any external stimulus and was gavage-fed. At 6 mo, she was referred to our hospital. She was treated with SB $(4 \mathrm{~g} / \mathrm{d})$, DX $(50 \mathrm{mg} / \mathrm{d})$, phenobarbital, diazepam, clonazepam, and vigabatrin. A reduction of the frequency of seizures was noticed. However, this treatment was not well tolerated, and the infant became agitated and inconsolable, which prompted DX withdrawal after 2 mo. Shortly thereafter, the neurologic status deteriorated with the appearance of a high-pitch cry and seizures. This patient did not respond to SB treatment and remained throughout her life hypotonic, lethargic, and unaware of her surroundings. Patients 3 and 4 had a similar course and died of an overwhelming form of $\mathrm{NKH}$ before any SB/DX treatment could be started. They both had a general increase in blood and CSF amino acids at birth. They were profoundly lethargic, with feeding difficulties and respiratory distress, which required respiratory support. In Patient 3 mild hyperammonemia was detected $(65.3 \mu \mathrm{M}$, normal $<35 \mu \mathrm{M})$. French ethics committees for research on patients approved the study, and parents provided written informed consent.

Serum and $\operatorname{CSF}^{1} \mathrm{H}$ MRS analysis. Paired samples of serum and CSF (unless otherwise stated) were analyzed. Lumbar punctures were performed for clinical reasons and not for the sole purpose of the current study. Samples were prepared following a method extensively described in a previous paper (12). Serum was preferred to plasma for ${ }^{1} \mathrm{H}$ MRS to eliminate signals from plasma proteins. ${ }^{1} \mathrm{H}$ MRS was performed at $400 \mathrm{MHz}$ on a Bruker AM 400 WB spectrometer (Wissembourg, France) equipped with a wide bore $9.4 \mathrm{~T}$ magnet and a quadronucleus $5-\mathrm{mm}$ probe. 3(trimethylsilyl)propionic-2,2,3,3- $d 4$ acid was used as an internal standard for chemical shift and concentration. Data were processed using the NMR1 software (New Methods Research Inc, Syracuse, NY). After zero-filling, apodization ( $\mathrm{LB}=0.5 \mathrm{~Hz}$ ), Fourier transform, phase, and baseline corrections spectra were integrated. Areas of overlapping signals were fitted using the NMR1 deconvolution procedure. For CSF analysis, chromatographic reference values were given in the absence of control values obtained by ${ }^{1} \mathrm{H}$ MRS for pediatric populations. Control values for organic acids are from the literature (13). Reference values for serum were obtained from a group of 15 children (age range, 2-15 y) who had been admitted to the hospital with a suspicion of neurologic disorder but in whom further examination

Table 1. Clinical and biochemical features of patients

\begin{tabular}{|c|c|c|c|c|c|}
\hline Variable & Patient 1 & Patient 2 & Patient 3 & Patient 4 & Reference values \\
\hline Sex & $\mathrm{F}$ & $\mathrm{F}$ & $\mathrm{F}$ & M & \\
\hline Family history & consanguinity & consanguinity & consanguinity & none & \\
\hline Hepatic GCS activity (a) & $2.9 \mathrm{nkat} / \mathrm{kg}$ & $2 \mathrm{nkat} / \mathrm{kg}$ & $2.4 \mathrm{nkat} / \mathrm{kg}$ & $1.6 \mathrm{nkat} / \mathrm{kg}$ & $117 \pm 80 \mathrm{nkat} / \mathrm{kg}$ \\
\hline CSF glycine (c) & $103 \mu \mathrm{M}$ & nd & $285 \mu \mathrm{M}$ & $288 \mu \mathrm{M}$ & $7 \pm 3 \mu \mathrm{M}$ \\
\hline CSF glycine/plasma glycine (d) & 0.11 & nd & 0.14 & 0.2 & $0.02-0.04$ \\
\hline CSF glutamine (e) & $436 \mu \mathrm{M}$ & nd & $437 \mu \mathrm{M}$ & $605 \mu \mathrm{M}$ & $580 \pm 50 \mathrm{mM}$ \\
\hline
\end{tabular}

From a to e, values at birth. Reference values are expressed as mean \pm SD. a, the control value is an average of 3-d measurement of GCS activity on a healthy population at hospital. Abbreviation used: nd, not determined. 
confirmed the absence of any neurologic pathology. A large-scale investigation of healthy neonates, infants, and children would be helpful to precisely define age-related metabolite concentrations in blood and CSF. However, such a study would not be ethically justifiable.

Chromatography. Ion-exchange chromatographic analysis of amino acids in CSF and blood plasma was performed as part of the clinical routine on a Beckmann AAA 6300 Gold analyzer (Paris, France).

MRI and localized ${ }^{1} \mathrm{H}$ MRS of brain. Examination of the patients was performed on a Siemens Magnetom SP63 equipped with a 1.5-T magnet (Erlangen, Germany). None of the children was sedated. Localization was performed on $T_{1^{-}}$weighted MR images and $T_{2}$-weighted MR images. Proton MRS (256 scans) was carried out using the STEAM sequence (stimulated echo acquisition mode) with $T E=20 \mathrm{~ms}, T M=30$ $\mathrm{ms}$ (mixing time), $T R=1500 \mathrm{~ms}$, and a volume of interest of $2 \times 2 \times 2 \mathrm{~cm}^{3}$ located in the occipitoparietal white matter. Short echo time sequences were selected to obtain the most informative spectra while minimizing discomfort for patients. Age is critical because ${ }^{1} \mathrm{H}$ MRS reflects the maturational changes occurring during the course of early brain development (14-16). Therefore, in the absence of infants in our control population, the age-related curves for cerebral metabolites published by Kreis et al. (15) were used to provide normal values from age-matched control subjects. This comparison was possible because similar methods and conditions were used (STEAM sequence performed in the parietal white matter, $T E=30 \mathrm{~ms}, T M=13 \mathrm{~ms}, T R=1500 \mathrm{~ms}$ ). Control spectra were also acquired from four healthy children (age range, 3-6 y). As a matter of fact, brain creatine reaches its definitive concentration about the age of $80 \mathrm{wk}$ GA and Ins-Gly about $130 \mathrm{wk}$ GA (15). Therefore, the brain spectra recorded from these four healthy controls are relevant for the interpretation of the results obtained on $\mathrm{tCr}$ and Ins-Gly levels after the age of $80 \mathrm{wk}$ and $130 \mathrm{wk}$ GA, respectively. Data were processed using the GIFA software (Centre de Biologie Structurale, Montpellier, France) and the IDL software (Interactive Data Language Research Systems, Boulder, CO, U.S.A.). Data processing consisted of zero-filling, gaussian filtering $(2 \mathrm{~Hz})$, Fourier transformation, zero-order phase correction, and manual baseline correction. The spectra were referenced to creatine (3.04 ppm). No line-fitting procedure was performed before peak integration. The signals stemming from Ins-Gly, Cho, creatine, and phosphocreatine ( $\mathrm{tCr}$ ), NAA, and Glx were integrated. Owing to technical limitations at the time of the MRS experiment, an aliasing of the lipid region was frequently observed on the spectrum and limited the accuracy of the determination of the brain lactate concentration. For Glx, the spectral regions corresponding to $\beta$ and $\gamma$ protons were integrated. Results were either expressed as ratios of the relative area of each metabolite signal to the sum of all metabolite signal areas (S) or as ratios of metabolites $(\mathrm{NAA} / \mathrm{tCr}, \mathrm{Cho} / \mathrm{tCr}$, and Ins-Gly/tCr). The evolution of these ratios was expressed as a function of GA to make comparison with the age-related normative curves published by Kreis et al. (15). GA refers to the age of the subject in weeks (including the weeks of gestation). Glx peak amplitude was significantly higher when using $T E=20 \mathrm{~ms}$; therefore no comparison was performed for the $\mathrm{Glx} / \mathrm{tCr}$ ratio. The integration values were not corrected for $T_{1}$ or $T_{2}(15)$.

\section{RESULTS}

Blood and CSF analysis in the absence of any SB/DX treatment. In addition to high levels of Gly in blood and CSF, the following observations were made. Among the four patients, two of them (patients 1 and 3) had an analysis of blood by ${ }^{1} \mathrm{H}$ MRS (Table 2). Hyperlactacidemia was detected in both cases. Creat and pyruvate were elevated in patient 1 but normal in patient 3 .

Table 2. Serum and CSF composition of patients 1-4 analyzed by 400-MHz ${ }^{1} \mathrm{H} M R S$

\begin{tabular}{|c|c|c|c|c|c|c|c|c|c|c|c|c|c|}
\hline & \multirow{3}{*}{$\begin{array}{c}\text { Normal } \\
\text { values }(\mu \mathrm{M})\end{array}$} & \multicolumn{6}{|c|}{ Patient 1} & \multicolumn{4}{|c|}{ Patient 2} & \multirow{2}{*}{\multicolumn{2}{|c|}{$\frac{\text { Patient } 3}{\text { Age of patients }}$}} \\
\hline & & \multicolumn{6}{|c|}{ Age of patient } & \multicolumn{4}{|c|}{ Age of patient } & & \\
\hline & & $10 \mathrm{mo}^{\mathrm{a}}$ & $13 \mathrm{mo}$ & $14 \mathrm{mo}$ & $18 \mathrm{mo}$ & $28 \mathrm{mo}$ & $6 \mathrm{y}$ & $7 \mathrm{mo}^{\mathrm{a}}$ & $8 \mathrm{mo}$ & $9 \mathrm{mo}$ & $15 \mathrm{mo}$ & $4 \mathrm{~d}^{\mathrm{a}}$ & $5 \mathrm{~d}^{\mathrm{a}}$ \\
\hline Glycine & $165 \pm 68$ & nd & 575 & 608 & 449 & 499 & 574 & nd & nd & nd & nd & 1337 & nd \\
\hline Creatine & $45 \pm 20$ & 80 & 81 & 108 & 76 & 65 & 158 & nd & nd & nd & nd & 31 & nd \\
\hline Citrate & $104 \pm 23$ & 139 & 97 & 108 & 137 & 65 & 34 & nd & nd & nd & nd & 28 & nd \\
\hline Succinate & $36 \pm 34$ & 57 & 45 & 60 & nd & nd & 15 & nd & nd & nd & nd & nd & nd \\
\hline Glutamine & $618 \pm 74$ & 830 & 435 & 539 & 388 & 556 & 594 & nd & nd & nd & nd & 357 & nd \\
\hline Alanine & $305 \pm 105$ & 524 & 257 & 315 & 277 & 438 & 460 & nd & nd & nd & nd & 124 & nd \\
\hline \multirow[t]{2}{*}{ Lactate } & $1655 \pm 279$ & 3633 & 3326 & 4616 & 4685 & 2229 & 1463 & nd & nd & nd & nd & 3132 & nd \\
\hline & & $10 \mathrm{mo}$ & $12 \mathrm{mo}$ & $14 \mathrm{mo}$ & $18 \mathrm{mo}$ & $23 \mathrm{mo}$ & $26 \mathrm{mo}$ & $7 \mathrm{mo}$ & $8 \mathrm{mo}$ & 9 mo & $15 \mathrm{mo}$ & $4 \mathrm{~d}$ & $5 \mathrm{~d}$ \\
\hline \multicolumn{14}{|l|}{$\mathrm{CSF}$} \\
\hline Glutamine & $580 \pm 50$ & 373 & 440 & 387 & 196 & 322 & 351 & 533 & 367 & 410 & 287 & nd & 556 \\
\hline Alanine & $25 \pm 9$ & 49 & 37 & 28 & 24 & 24 & 19 & 20 & 44 & 27 & 26 & nd & 42 \\
\hline Lactate & $1390 \pm 750$ & 2304 & 4089 & 2532 & 1746 & 2528 & 1693 & 2259 & 2195 & 2859 & 2298 & nd & 1412 \\
\hline
\end{tabular}

${ }^{\text {a }}$ Analyses performed before the onset of SB/DX treatment. Reference values are expressed as mean \pm SD. nd, not determined. 
The general increase in blood and CSF alanine observed at birth was still present in patient 1 at $10 \mathrm{mo}(83 \mathrm{wk} \mathrm{GA}$, Tables 2 and 3 ). In patient 2, blood chromatography evidenced an increase in cysteine. Three patients (patients 1,2, and 4) had an analysis of CSF by ${ }^{1} \mathrm{H}$ MRS. A typical ${ }^{1} \mathrm{H}$ MRS spectrum of CSF is shown in Figure 1. A major reduction of CSF citrate was noticed in all cases (Table 2). CSF glutamine was below the normal range in patients 1 and 3 (Tables 1 and 2). In addition, patients 1 and 2 had an increased concentration of lactate in CSF whereas a high level of pyruvate was detected in patient 1 only.

Blood and CSF analysis after institution of SB/DX treatment. In patient 1 , blood chromatography and ${ }^{1} \mathrm{H}$ MRS of serum showed that Gly concentration had been lowered on treatment, although it was still above the normal range (Tables 2 and 3). A reversed tendency was observed at $53 \mathrm{mo}$ (270 wk GA), when a rise in Gly was detected in spite of an increasing dosage of SB/DX. The hyperaminoacidemia observed at birth was reduced on treatment until the age of 6 (353 wk GA), when an increase in all amino acids occurred. From the age of 31 mo (174 wk GA), Glx were measured by chromatography as a pair of metabolites; however, ${ }^{1} \mathrm{H}$ MRS of serum showed that glutamine was generally within the normal range. Lactate and creatine (Table 2) were frequently high. Pyruvate normalized instantly on treatment. The analysis of CSF revealed that the decrease observed in plasma Gly was paralleled by a slight reduction in CSF Gly as observed by chromatography. ${ }^{1} \mathrm{H}$ MRS results were inconclusive in that regard. CSF glutamine was often low as observed by chromatography and ${ }^{1} \mathrm{H}$ MRS, whereas high concentrations in proline and alanine were persistent. Pyruvate concentration, which was high in the first

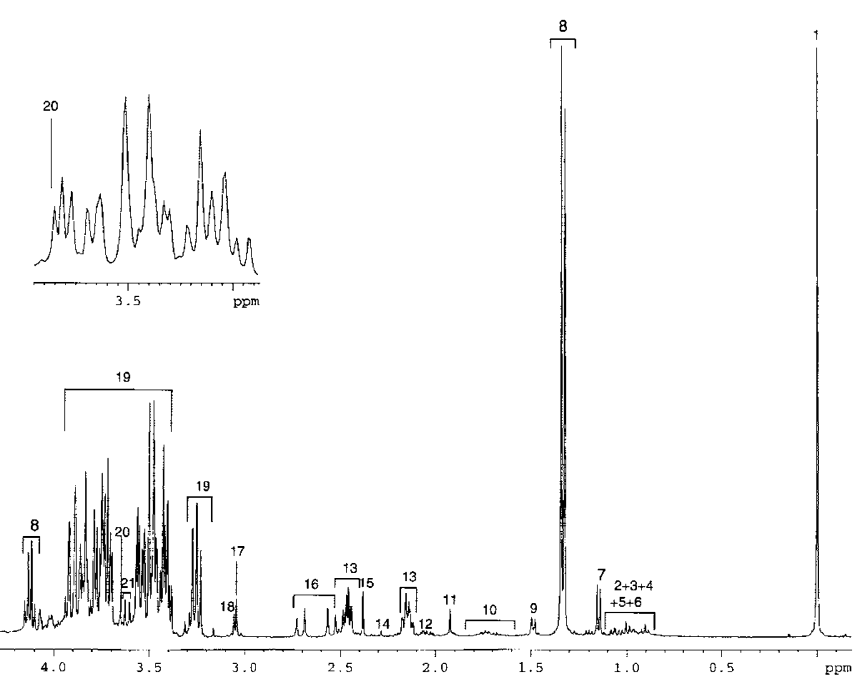

Figure 1. $400-\mathrm{MHz}{ }^{1} \mathrm{H}-\mathrm{MRS}$ spectrum of CSF. The analysis was performed on the CSF of patient 2 at the age of 8 mo. The spectrum shows a high level of Gly, elevated concentrations of pyruvate and alanine, reduced contents of citrate and glutamine. Inset, expansion of the region containing Gly. Assignations: 1, (trimethylsilyl)propionic-2,2,3,3- $d 4$ acid; 2, $\alpha$-hydroxybutyrate; 3, $\alpha$-aminobutyrate; 4 , leucine; 5 , isoleucine; 6 , valine; $7, \beta$-hydroxybutyrate; 8 , lactate; 9, alanine; 10, lysine; 11, acetate; 12, $\mathrm{N}$-acetyl compounds; 13 , glutamine; 14 , acetone; 15 , pyruvate; 16 , citrate; 17 , creatine; 18 , creatinine; 19, glucose; 20, glycine; 21, myo-inositol. 3(trimethylsilyl)propionic$2,2,3,3-d 4$ acid was used as an internal standard for chemical shift and concentration. years of therapy, normalized with increasing dosage of SB/DX, but citrate remained low. In patient 2, blood chromatography showed a dramatic decrease in Gly concentration at the end of life whereas cysteine, proline, and alanine were significantly elevated (Table 3). Chromatography and ${ }^{1} \mathrm{H}$ MRS of CSF showed that from 7 to 9 mo (70 to 78 wk GA), Gly concentration increased considerably although plasma Gly dropped, which confirmed that patient 2 was rather unresponsive to treatment (Tables 2 and 3). Glutamine was often reduced and citrate was extremely low, whereas proline, alanine, and creatine were increased.

MRI of brain. The $T_{1}$-weighted images recorded for patient 1 demonstrate a thin corpus callosum (Fig. 2A). Cortical and subcortical atrophy were detected on axial $T_{2}$-weighted images (Fig. 2B). Interestingly, axial $T_{2}$-weighted images show a reduction of the cortical atrophy with time and treatment (Fig. 2 , bottom) and delayed but progressive myelination. The improvement of the cortical atrophy is characterized by a slight reduction in the size of the lateral ventricles and a clear reduction in the thickness of the sulci. In the case of patient 2 , a severe dysgenesis of the corpus callosum was detected on coronal and sagittal $T_{I}$-weighted images (Fig. 3, top). The long-term follow-up of this patient demonstrated no progression of the cortical atrophy that was noticed on the first axial $T_{2}$-weighted MR image (Fig. 3, bottom). Moreover, there was no progression of brain myelination during this follow-up.

Localized ${ }^{1} \boldsymbol{H}$ MRS of brain. The results are expressed as peak area ratios that are representative of concentration ratios, but can under certain conditions be modified by relaxation times. Figure 4 shows the evolution of brain metabolites with age and treatment in patients 1 and 2 . Table 4 provides age-matched comparison for Ins-Gly/tCr, NAA/tCr, and Cho/ tCr. In patient 1 , Ins-Gly/tCr was significantly higher than normal at 79 wk GA $(+70 \%$ compared with age-matched control subjects) but regained normal value soon after the institution of $\mathrm{SB} / \mathrm{DX}$ treatment. $\mathrm{NAA} / \mathrm{tCr}$ was always within the normal range when compared with the control values from the literature. Cho/tCr was variable but tended to decrease and was lower than the age-matched control value at the age of $6 \mathrm{y}$ (353 wk GA; Table 4). The evolution of brain metabolite profile displayed in Figure 4 and Table 4 shows a reduction of Ins-Gly/S and a concomitant increase of all metabolites after the institution of SB/DX treatment. However, Ins-Gly/S regained its initial value by the age of $353 \mathrm{wk}$ GA. Cho/S was occasionally low, whereas $\mathrm{tCr} / \mathrm{S}$ and NAA/S increased progressively (Table 4). At the age of 6 y (353 wk GA), Ins-Gly/S, $\mathrm{Cho} / \mathrm{S}$, and NAA/S were within the normal range whereas $\mathrm{tCr} / \mathrm{S}$ was slightly higher than in age-matched control subjects (Fig. 4). The NAA/Ins-Gly ratio, which was extremely low before the onset of therapy, recovered thereafter and remained close to normal (Fig. 5). In patient 2 a different metabolic pattern was observed. The Ins-Gly/tCr ratio was always higher than normal. At the age of 7 mo (70 wk GA), this ratio was $70 \%$ higher than the reference value. $\mathrm{NAA} / \mathrm{tCr}$ decreased to subnormal values with age and treatment; $\mathrm{Cho} / \mathrm{tCr}$, although variable, was within the normal range (Table 4). Figure 4 shows that Ins-Gly/S was significantly higher than in patient 1. This ratio declined with increasing age and treatment. A tem- 
Table 3. Amino acid concentrations in plasma and CSF measured by chromatography for patients 1 and 2

\begin{tabular}{|c|c|c|c|c|c|c|c|c|c|c|c|}
\hline & \multirow{3}{*}{$\begin{array}{c}\text { Reference } \\
\text { values }(\mu \mathrm{M})\end{array}$} & \multicolumn{5}{|c|}{ Patient 1} & \multicolumn{5}{|c|}{ Patient 2} \\
\hline & & \multicolumn{5}{|c|}{ Age of patient } & \multicolumn{5}{|c|}{ Age of patient } \\
\hline & & $10 \mathrm{mo}^{\mathrm{a}}$ & $14 \mathrm{mo}$ & $31 \mathrm{mo}$ & $53 \mathrm{mo}$ & $6 y$ & $7 \mathrm{mo}^{\mathrm{a}}$ & $8 \mathrm{mo}$ & 9 mo & $15 \mathrm{mo}$ & $19 \mathrm{mo}$ \\
\hline Glycine & $200 \pm 40$ & 817 & 679 & 278 & 525 & 732 & 893 & 512 & 413 & 80 & 150 \\
\hline Glutamate & $90 \pm 65$ & 55 & 43 & $443 *(465-609)$ & $580 *$ & $773 *$ & 86 & 87 & 199 & $682 *$ & $670^{*}$ \\
\hline Glutamine & $580 \pm 180$ & 782 & 406 & $443 *$ & $580^{*}$ & $773 *$ & 733 & 613 & 388 & $682 *$ & $670^{*}$ \\
\hline Proline & $164 \pm 71$ & 122 & 133 & 107 & 169 & 327 & 183 & 329 & 381 & 317 & 567 \\
\hline \multicolumn{12}{|l|}{$\mathrm{CSF}$} \\
\hline Glycine & $7 \pm 3$ & 55 & 50 & 44 & nd & nd & 122 & 170 & 164 & nd & nd \\
\hline Glutamine & $580 \pm 50$ & 428 & 296 & $369 *(355-855)$ & nd & nd & 387 & 525 & 441 & nd & nd \\
\hline Proline & $0.4 \pm 0.3$ & 3 & nd & nd & nd & nd & nd & 4 & 3 & nd & nd \\
\hline Alanine & $25 \pm 9$ & 38 & 20 & 24 & nd & nd & 21 & 55 & 47 & nd & nd \\
\hline
\end{tabular}

${ }^{a}$ Analyses performed before the onset of SB/DX treatment. Reference values from age-matched controls are presented in parentheses. * Value common to glutamine and glutamate. Reference values are expressed as mean \pm SD. nd, not determined.
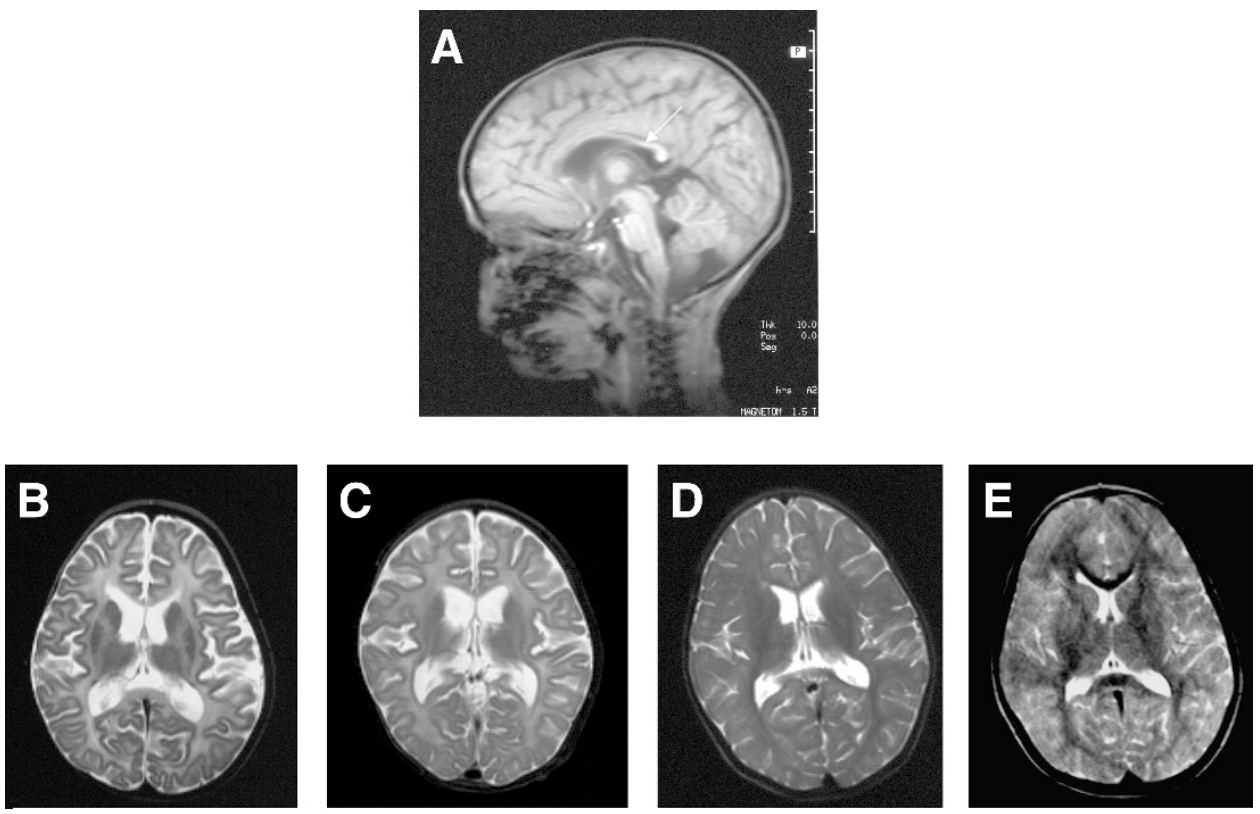

Figure 2. Brain MRI images of patient 1. Sagittal $T_{1}$-weighted MR image $(A)$ discloses a thin corpus callosum. Axial $T_{2}$-weighted MR images sectioned at the same level (bottom) show an improvement of the cortical atrophy characterized by a slight reduction in the size of the lateral ventricles and a clear reduction in the thickness of the sulci. $A$ was recorded at the age of $16 \mathrm{mo}(T R / T E, 200 / 10 \mathrm{~ms}) . B-D$ were recorded at 10,12 , and $23 \mathrm{mo}$, respectively $(T R / T E, 3500 / 120$ $\mathrm{ms}) . E$ was obtained at the age of 6 y (TR/TE, 4200/90 ms).

porary rise in all metabolites was noticed after the institution of $\mathrm{SB} / \mathrm{DX}$ regimen. There was a marked trend for $\mathrm{tCr} / \mathrm{S}$ to be raised to significantly higher levels than those observed for patient 1 . Another difference with patient 1 is the low level of NAA, which was not enhanced with age or treatment. The Glx content was extremely variable, although significantly lower than in patient 1 . A temporary rise of Glx/S was observed after the institution of SB/DX, immediately followed by a fall as in patient 1 . The NAA/Ins-Gly ratio remained dramatically low throughout the disease in comparison with age-matched control subjects and patient 1 (Fig. 5).

\section{DISCUSSION}

The aim of this study was to identify, by using ${ }^{1} \mathrm{H}$ MRS, abnormalities other than increased Gly in blood and CSF of
$\mathrm{NKH}$ patients and to investigate alterations of brain metabolism in these patients. The underlying purpose of this approach was to test whether metabolic abnormalities in fluids could be related to a specific cerebral metabolite pattern. Another objective was to define metabolic clues that may have a role in the assessment of the outcome of NKH patients.

Analysis of blood and CSF. The results obtained by ${ }^{1} \mathrm{H}$ MRS of serum were generally in good agreement with the chromatographic analysis, which was performed in parallel. ${ }^{1} \mathrm{H}$ MRS was sensitive in detecting metabolite changes in blood and CSF, although some slight discrepancies were noticed when estimating Gly concentration. These differences could stem from an overlap between Gly, Ins, and glucose resonances. Moreover, blood plasma was analyzed by chromatography whereas ultrafiltered blood serum was analyzed by ${ }^{1} \mathrm{H}$ 

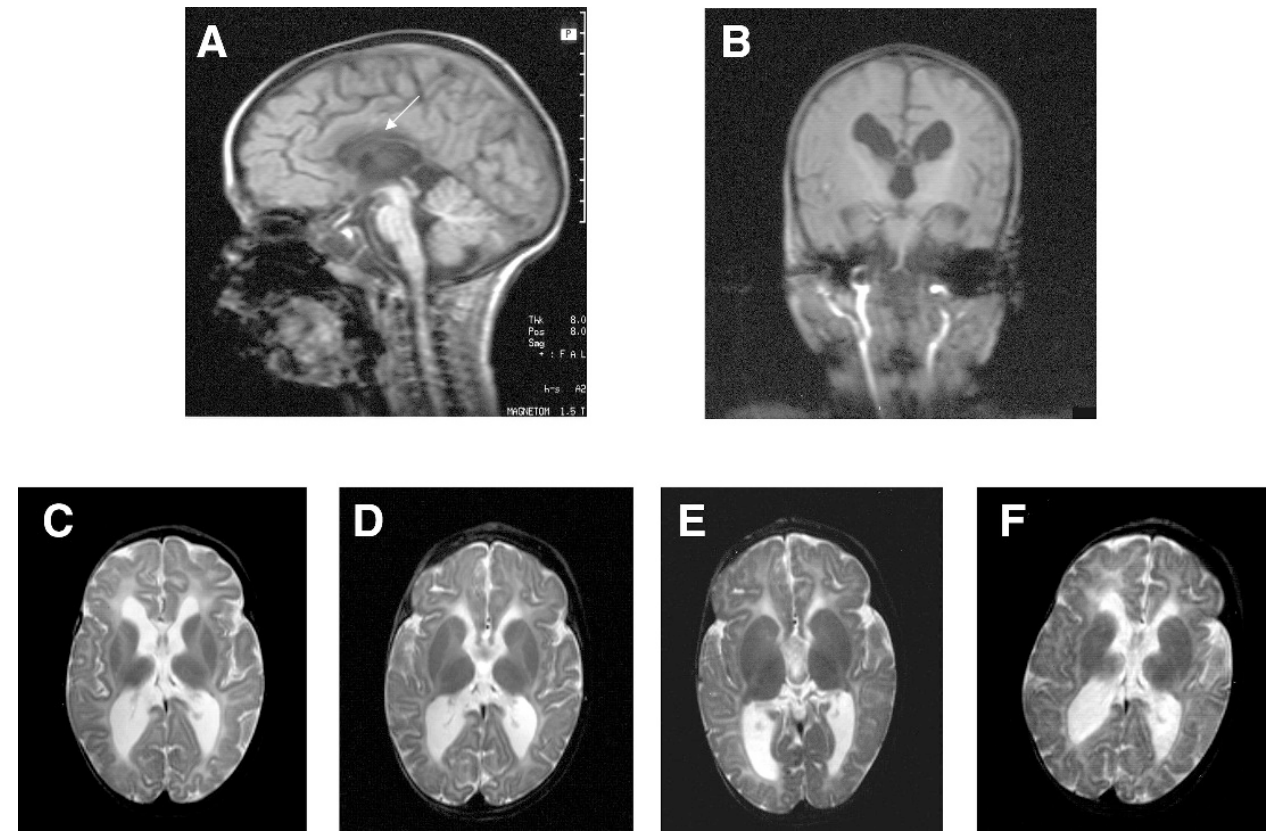

Figure 3. Brain MRI images of patient 2. Sagittal $(A)$ and coronal $(B) T_{1}$-weighted MR images evidence a severe dysgenesis of the corpus callosum. Axial $T_{2}$-weighted MR images sectioned at the same level (bottom) do not show any progression of the cortical atrophy or evolution in brain myelination. No significant differences were observed. $A$ and $B$ were recorded at the age of 9 mo (TR/TE, 350/10 ms). $C-F$ were recorded at 7, 9, 13, and 15 mo, respectively (TR/TE, $3500 / 120 \mathrm{~ms})$.

MRS. This difference may also generate variations in the amino acid concentrations. There is a long-standing observation of general increase in blood and CSF amino acids in NKH $(2,17)$, albeit not fully exploited. Although Gly biochemistry is well known, these alterations were never given any explanation. Our MRS study of blood and CSF indicates that these deviations can be ascribed to Gly disposal mechanisms and that at least two pathways, essentially linked to L-arginine glycine amidinotransferase (EC 2.1.4.1) and serine hydroxymethylase (EC 2.1.2.1) activities, coexist (Fig. 6, steps 2 and 4). Lactic acidosis is common in a number of conditions including metabolic inborn errors. We have found high levels of lactate in plasma and CSF of NKH patients, but the cause of this excess of lactate has remained unclear $(18,19)$. We suggest it could result from Gly conversion into pyruvate (Fig. 6). Under normal circumstances, the conversion of serine to Gly is favored. In NKH, high levels of Gly require catabolism, and Gly conversion to serine is a way to reduce Gly. Moreover, patients usually undergo dietary restriction in serine, which necessitates endogenous synthesis from the Gly pool. One end point of this pathway is lactate formation (Fig. 6). As a matter of fact, other intermediates of this pathway were elevated in all patients, inasmuch as in addition to lactate and serine, high levels of alanine, proline, and pyruvate have been detected. High levels of alanine and proline were persistent in spite of SB/DX treatment. Lactic acidosis associated with hyperalaninemia and hyperprolinemia has been described in a number of metabolic disorders $(20,21)$. Excess of alanine originates from lactate conversion to pyruvate and its subsequent transamination into alanine (Fig. 6, step 5), whereas proline accumulation could result from proline oxidase (EC 1.5.99.8) inhibition by lactate (22) (Fig. 6, step 7). We also observed an
PATIENT 1
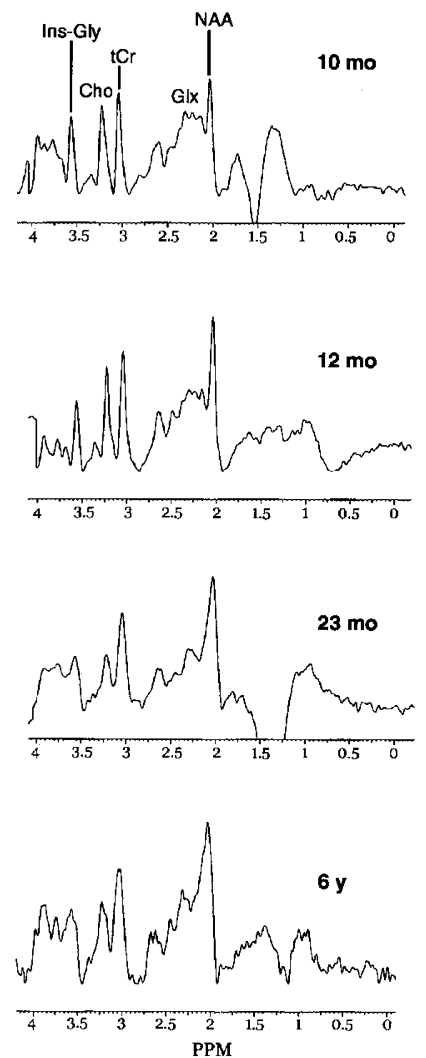

PATIENT 2
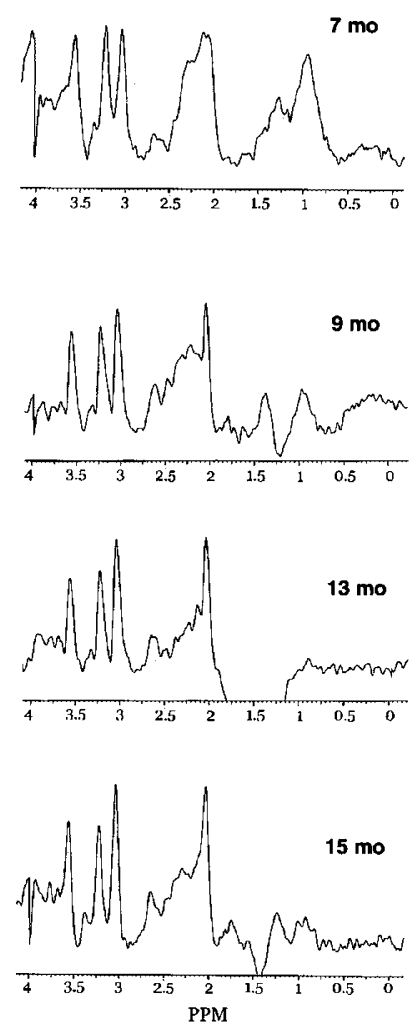

Figure 4. ${ }^{1} \mathrm{H}$ MRS brain spectra of patients 1 (left) and 2 (right). Proton spectra were recorded from the occipitoparietal white matter at $T E=20$ $\mathrm{ms}$. The first spectra at $10 \mathrm{mo}(83 \mathrm{wk} \mathrm{GA})$ in patient 1 and at $7 \mathrm{mo}(70 \mathrm{wk}$ GA) in patient 2 were the only ones performed before the onset of SB/DX treatment. 
VIOLA ET AL.

Table 4. Evolution of cerebral metabolites ratios obtained by in vivo ${ }^{I} H$ MRS in patients 1 and 2

\begin{tabular}{|c|c|c|c|c|c|c|c|c|}
\hline \multirow{3}{*}{$\begin{array}{c}\text { PNA } \\
\text { GA (wk) }\end{array}$} & \multicolumn{4}{|c|}{ Patient 1} & \multicolumn{4}{|c|}{ Patient 2} \\
\hline & $10 \mathrm{mo}^{\mathrm{a}}$ & $12 \mathrm{mo}$ & $23 \mathrm{mo}$ & $6 y$ & $7 \mathrm{mo}^{\mathrm{a}}$ & $9 \mathrm{mo}$ & $13 \mathrm{mo}$ & $15 \mathrm{mo}$ \\
\hline & 83 & 91 & 139 & 353 & 70 & 78 & 96 & 104 \\
\hline Cho/S & 0.15 & 0.17 & 0.12 & 0.16 & 0.16 & 0.21 & 0.18 & 0.17 \\
\hline $\mathrm{tCr} / \mathrm{S}$ & $0.13(0.12 \pm 0.02)$ & $0.19(0.12 \pm 0.02)$ & $0.2(0.12 \pm 0.02)$ & $0.21(0.12 \pm 0.02)$ & 0.17 & 0.23 & $0.24(0.12 \pm 0.02)$ & $.2(0.12 \pm 0.02)$ \\
\hline $\mathrm{NAA} / \mathrm{S}$ & 0.19 & 0.25 & 0.27 & 0.31 & 0.22 & 0.23 & 0.27 & 0.23 \\
\hline $\mathrm{Glx} / \mathrm{S}$ & 0.55 & 0.63 & 0.37 & 0.44 & 0.32 & 0.54 & 0.30 & 0.23 \\
\hline $\mathrm{NAA} / \mathrm{tCr}$ & $1.44(1.20)$ & $1.29(1.30)$ & $1.37(1.40)$ & $1.42(1.44)$ & $1.28(1.20)$ & $0.98(1.27)$ & $1.11(1.30)$ & $1.15(1.30)$ \\
\hline
\end{tabular}

The control values (in parentheses) are from Kreis et al. 15 . Control values of $\mathrm{tCr} / \mathrm{S}(0.12 \pm 0.02)$ and Ins-Gly/S $(0.17 \pm 0.03)$ are from four control subjects (age range, 3-6 y, 196-353 wk GA). a Analyses performed before the onset of SB/DX treatment. PNA, postnatal age.

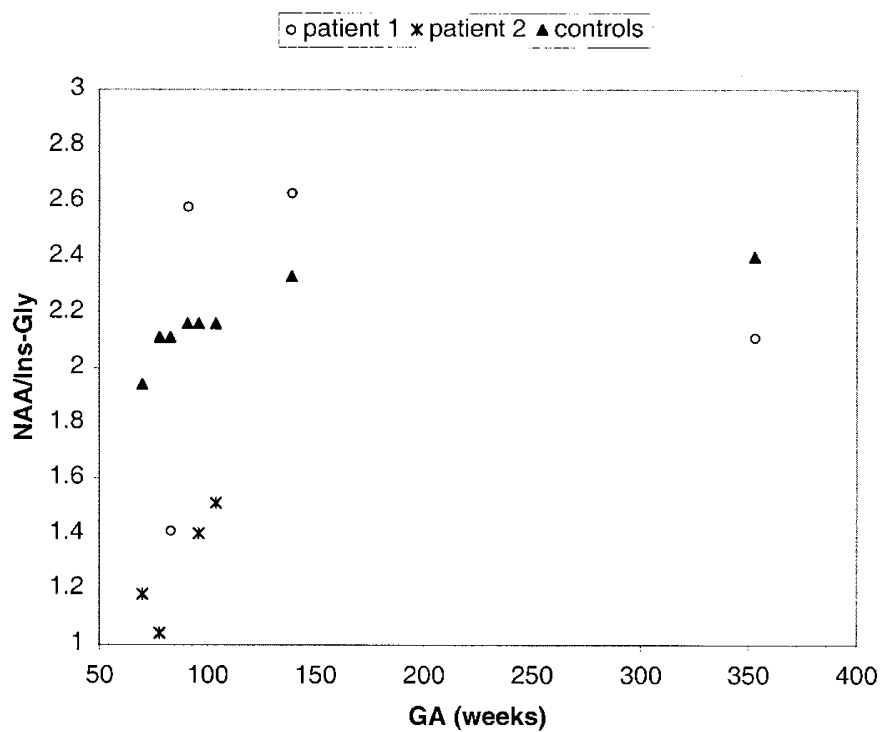

Figure 5. Evolution of the brain NAA/Ins-Gly ratio in patients 1 and 2 . Control values for NAA/Ins-Gly are from literature (15). The first data points at $10 \mathrm{mo}(83 \mathrm{wk} \mathrm{GA})$ in patient 1 and at $7 \mathrm{mo}(70 \mathrm{wk} \mathrm{GA})$ in patient 2 were the only ones performed before the onset of SB/DX treatment.

increase in plasma cysteine, which could be related to serine hydroxymethylase activity (23) (Fig. 6). Taken together, these results indicate that high levels of lactate, pyruvate, alanine, proline, and sulfur amino acids originate from Gly disposal via the action of serine hydroxymethylase. Increased levels of creatine were detected by ${ }^{1} \mathrm{H}$ MRS in blood and CSF when Gly concentrations were extremely high. This elevation could also result from Gly disposal mechanisms (Fig. 6) (24). Another frequent feature in NKH patients is the reduced content of glutamine in CSF. Plasma glutamine, which is lowered in a number of conditions (25-27) by activation of a catabolic pathway or reduction in synthesis, was normal in NKH patients. The level of glutamine in CSF probably reflects changes in cerebral metabolism of glutamine. A hypothesis to explain this decrease could rely on a partial inhibition of brain GS (EC 6.3.1.2) possibly by Gly (Fig. 6, step 8), as it has been shown that this amino acid can inhibit GS $(28,29)$. Finally, the recurrent observation of a dramatic collapse in CSF citrate could indicate a dysfunction of the tricarboxylic acid cycle, which is linked to glutamine metabolism in brain (Fig. 6, inset).
MRS of brain. At birth, Cho and Ins-Gly are the dominant metabolites on the brain proton spectrum. The most evident maturational changes beginning in the perinatal period is the continued increase in the NAA signal relative to the Cho and tCr signals. By approximately 6 mo of age, NAA becomes the major peak and its increase is nearly complete by early childhood (2-5 y) (15). The longitudinal follow-up of patients 1 and 2 using ${ }^{1} \mathrm{H}$ MRS of brain allowed us to detect these maturational changes. However, the brain patterns of patients 1 and 2 also showed signs of abnormal brain metabolism, which was more pronounced in patient 2. Patient 1 seemed to be affected by a milder form of NKH whereas patient 2 had a fatal course. In patient 1 , Ins- $\mathrm{Gly} / \mathrm{tCr}$ was significantly higher than the control value before treatment. After the institution of SB/DX treatment, Ins-Gly/S decreased. This fall is partly ascribed to the expected reduction of Ins on aging. Ins is a glial marker, and several cases of gliosis have been reported. Therefore, a reduction of Ins could also reflect an improvement at the level of glial cells as a consequence of the treatment. But previous MRS studies on NKH infants, using a longer spin echo time, have demonstrated that Gly is the main contributor to the signal arising at $3.57 \mathrm{ppm}$ (9). Consequently, the normalization of the Ins-Gly signal could reflect a fall of Gly itself as there was a concomitant reduction of Gly in blood and to a lesser extent in CSF. A rapid recovery of the NAA signal and a normalization of the NAA/Ins-Gly ratio occurred at $12 \mathrm{mo}$ (91 wk GA). These changes indicate an improvement in neuronal development. Cho/tCr decreased with time and treatment whereas $\mathrm{Cho} / \mathrm{S}$ showed a trend to increase. This result is in contrast with the expected decline of Cho owing to brain maturation and could reflect abnormal myelination. In patient 2 , in parallel to the rise of Gly in CSF, an increase of the Ins-Gly signal was observed in brain. This signal remained dramatically elevated although decreasing toward the age of 13 mo (96 wk GA). The Ins-Gly/tCr was not reduced with age when the contribution of Ins to the signal should be diminished. The higher level of brain Ins-Gly is probably linked to the severe clinical course of this patient. The level of NAA was dramatically low in this child, which is a sign of major neurodevelopmental impairment.

A progressive and persistent increase in $\mathrm{tCr}$ was observed in both patients 1 and 2 . The difficulty in interpreting this result relies on several factors: 1) there is a physiologic increase of creatine concentration in the occipitoparietal white matter until 


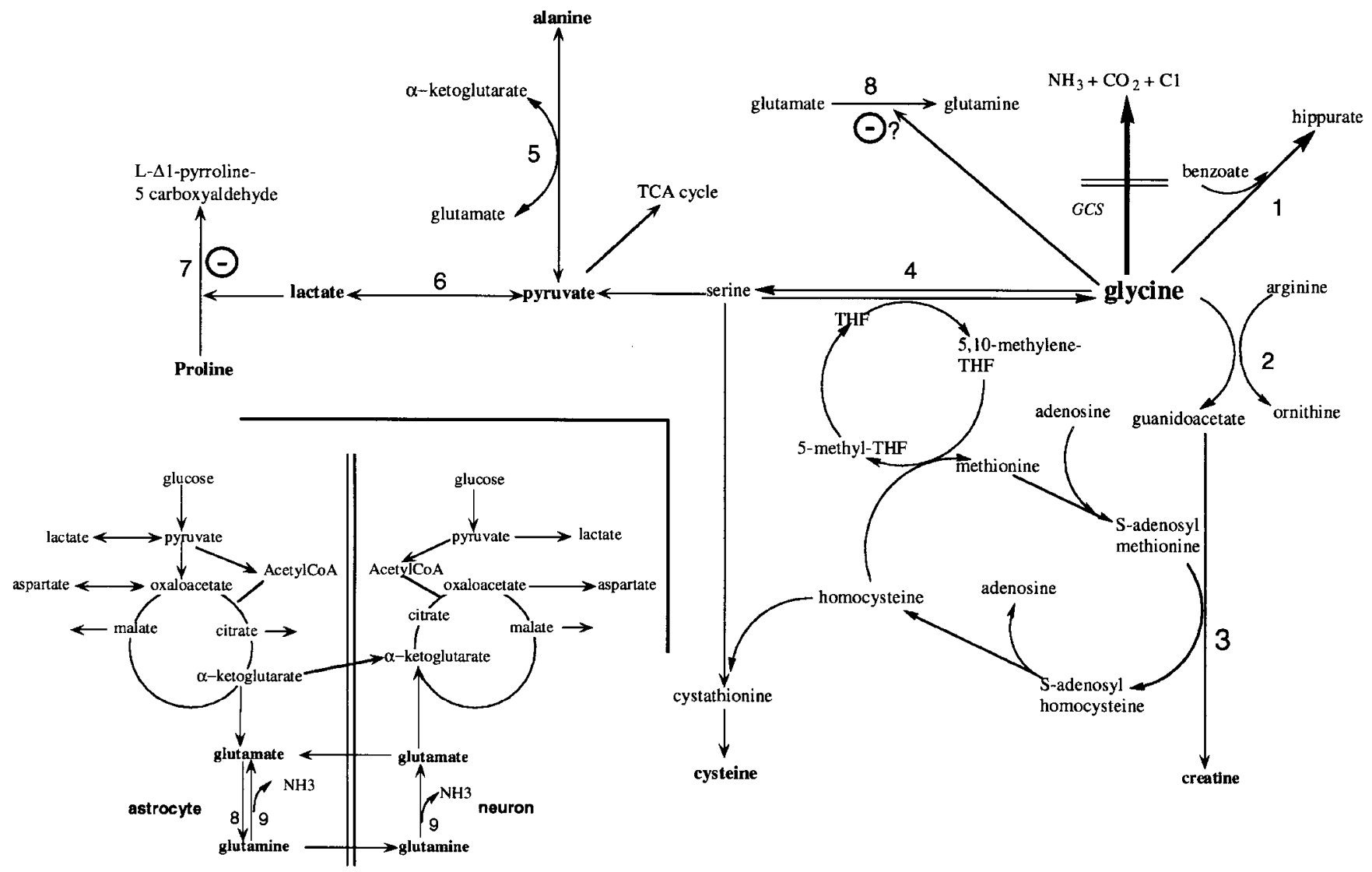

Figure 6. Gly metabolic pathways in NKH. 1, glycine acyltransferase (EC 2.3.1.13); 2, L-arginine:glycine amidinotransferase (EC 2.1.4.1); 3, guanidinoacetate $N$-methyltransferase (EC 2.1.1.2); 4, serine hydroxymethylase (EC 2.1.2.1); 5, alanine aminotransferase (EC 2.6.1.2); 6, lactate dehydrogenase (EC 1.1.1.27); 7, proline oxidase (EC 1.5.99.8); 8, glutamine synthetase (EC 6.3.1.2). THF, tetrahydrofolate. Inset, glutamine/glutamate cycle in neurons and astrocytes. 9, phosphate-activated glutaminase (EC 3.5.1.2).

the age of 80 wk GA (15);2) creatine is a putative marker of gliosis that is a frequent feature of NKH disease; 3) creatine is produced on Gly conversion; 4) gliosis is associated with increased creatine and Ins, but Ins is raised in proton brain spectra of NKH patients because of the resonance of Gly; and 5) there is a physiologic decrease of Ins concentration (until approximately the age of $130 \mathrm{wk}$ GA) in occipitoparietal white matter with maturation (15). Taking into account all these possibilities, we suggest that the increase in brain creatine observed in both patients 1 and 2 is not correlated with brain maturation or gliosis. In patient 1 , a sustained decrease of Ins-Gly is observed until the age of $6 \mathrm{y}$, whereas creatine continues to increase and reaches abnormally high levels. This finding is not compatible with gliosis. Moreover, MRI images show an improvement of the cortical atrophy. Patient 2, who suffered from a lethal form of the disease, displayed higher concentrations of brain Ins-Gly and creatine (Table 4), but as in the case of patient 1 , MRI did not show any progression of the cortical atrophy. Finally, given the increase of creatine in body fluids, the augmentation of brain creatine appears to be part of a diffuse metabolic problem. It is likely to reflect a disposal pathway for Gly as previously described for blood and CSF. Increased CSF creatine was observed in patient 2 before and after treatment but not anymore in patient 1 after the institution of SB/DX treatment. As creatine synthesis by glial cells was demonstrated recently (30), it is highly probable that elevated brain creatine results from the endogenous conversion of brain Gly to creatine. In view of the improvement of the NAA content with time in patient 1 , the marked trend for brain $\mathrm{tCr}$ to increase might be a sign of recovery. Interestingly, the fact that $\mathrm{tCr}$ reached a higher concentration in patient 1 than in agematched control subjects, in spite of an Ins-Gly/S ratio within the normal range, could ultimately confirm the hypothesis of Gly recycling to $\mathrm{tCr}$.

In both patients strong variations in the signals stemming from Glx were observed. In patient 1 , Glx signals were elevated in early infancy but decreased thereafter to reach a value within the normal range at the age of 6 y ( $353 \mathrm{wk}$ GA). In patient 2, Glx signals were much lower than in patient 1 , except at the age of $9 \mathrm{mo}$ (78 wk GA), when a level of Glx similar to that observed in patient 1 at the age of 10 mo ( 83 wk GA) was noticed. A significant increase in Glx has already been reported in a case of NKH (11). Interestingly, an inverse correlation between NAA recovery and Glx reduction was observed in patient 1. In patient 2, brain Glx dropped at the age of 13 mo (96 wk GA) as almost all brain metabolites did, including NAA, which is the sign of pronounced cellular dysfunction possibly leading to neuronal loss. Finally, the low level of glutamine observed in the CSF of both patients could suggest some abnormality in the brain glutamine metabolism, such as 
the hypothesis of brain GS inhibition by Gly. This assumption raises the possibility of a diminished supply of glial glutamine to neurons that could result in neuroexcitotoxic injury and impairment of energy production in neurons $(31,32)$ (Fig. 6). This hypothesis could explain the marked deficit observed in CSF citrate and partly underlie the neuronal damage observed in these patients and the astrocytic gliosis and neuronal loss described at autopsy in several cases $(2,33,34)$.

Biochemical markers for the severity of the disease. The lethal form of NKH appeared to be associated with elevated levels of Ins-Gly in brain, high creatine, and reduced NAA. There was a great difference between patient 1 , who is affected by a milder form of the disease, and patient 2 , who had an adverse course. This was remarkably clear when considering the evolution of NAA/Ins-Gly. Comparative analysis of brain spectral profiles and clinical courses indicated that a low NAA/Ins-Gly ratio carries a poor prognosis.

In conclusion, this study, which aimed at clarifying the biochemistry underlying the pathophysiology of NKH, allowed the identification of pathways for Gly disposal. Although Gly biochemistry is well known, amino acid deviations in NKH have never been thoroughly investigated. In this study we have shown that NKH is associated with increased concentrations of lactate, alanine, proline, and creatine in fluids and a significant elevation of brain creatine as a probable consequence of Gly disposal. The elevation of brain creatine in NKH disease is an absolutely new finding. The identification of NAA/Ins-Gly as an indicator for the severity of the disease could be ultimately applied to monitor and to predict the evolution of NKH and fine-tune the existing and future treatments. In view of the findings of the present study, the combined in vitro and in vivo MRS techniques may play a significant role in the assessment of adverse outcome in NKH disease.

Acknowledgments. The authors thank Dr. J. Vion-Dury for his initial interest in this work, Dr. E. Cabanes for assistance in data processing, Dr. N.W. Lutz for advice in the analysis of spectroscopic results, and Prof. J. Mancini and Dr. F. Vintila for their clinical collaboration.

\section{REFERENCES}

1. Hamosh A, Johnston MV, Valle D 1995 Non-ketotic hyperglycinemia. In: Scriver CR, Beaudet AL, Sly WS, Valle D (eds) The metabolic basis of inherited diseases. McGraw-Hill, New York, pp 1337-1348

2. Langan TJ, Pueschel SM 1983 Non-ketotic hyperglycinemia: clinical, biochemical, and therapeutic considerations. Curr Probl Pediatr 13:1-30

3. Perry TL, Urquhart N, Mac Lean J, Evans ME, Hansen S, Davidson GF, Applegarth DA, Mac Leod PJ, Lock JE 1975 Non-ketotic hyperglycinemia: glycine accumulation due to absence of glycine cleavage in brain. N Engl J Med 292:1269-1273

4. Steiner RD, Sweetser DA, Rohrbaugh JR, Dowton SB, Toone JR, Applegarth DA 1996 Non-ketotic hyperglycinemia: atypical clinical and biochemical manifestations. J Pediatr 128:243-246

5. Kure S, Tada K, Narisawa K 1997 Non-ketotic hyperglycinemia: biochemical, molecular and neurological aspects. Jpn J Hum Genet 42:13-22
6. Van Hove JLK, Kishnani PS, Demaerel P, Kahler SG, Miller C, Jaeken J, Rutledge SL 2000 Acute hydrocephalus in non-ketotic hyperglycinemia. Neurology 54:754756

7. Brun A, Borjeson M, Hultberg B, Sjoblad S, Akesson H, Litwin E 1979 Neonatal non-ketotic hyperglycinemia: a clinical, biochemical and neuropathological study including electron microscopic findings. Neuropaediatrie 10:195-205

8. Shuman RM, Leech RW, Scott CR 1978 The neuropathology of the non-ketotic and ketotic hyperglycinemias: three cases. Neurology 28:139-146

9. Heindel W, Kugel H, Roth B 1993 Non-invasive detection of increased glycine content by proton MR spectroscopy in the brains of two infants with non-ketotic hyperglycinemia. AJNR Am J Neuroradiol 14:629-635

10. Iwama H, Takahashi K, Kure S, Hayashi F, Narisawa K, Tada K, Mizoguchi M, Takashima S, Tomita U, Nishikawa T 1997 Depletion of cerebral D-serine in non-ketotic hyperglycinemia: possible involvement of glycine cleavage system in control of endogenous D-serine. Biochem Biophys Res Commun 231:793-796

11. Gabis L, Parton P, Roche P, Lenn N, Tudorica A, Huang W 2001 In vivo ${ }^{1} \mathrm{H}$ magnetic resonance spectroscopic measurement of brain glycine levels in non-ketotic hyperglycinemia. J Neuroimaging 11:209-211

12. Maillet S, Vion-Dury J, Confort-Gouny S, Nicoli F, Lutz NW, Viout P, Cozzone PJ 1998 Experimental protocol for clinical analysis of cerebrospinal fluid by high resolution proton magnetic resonance spectroscopy. Brain Res Brain Res Protoc 3:123-134

13. Hoffmann GF, Seppel CK, Holmes B, Mitchell L, Christen HJ, Hanefeld F, Rating D, Nyhan WL 1993 Quantitative organic acid analysis in cerebrospinal fluid and plasma: reference values in a pediatric population. J Chromatogr B Biomed Appl 617:1-10

14. Huppi PS, Barnes PD 1997 Magnetic resonance techniques in the evaluation of the newborn brain. Clin Perinatol 24:693-723

15. Kreis R, Ernst T, Ross BD 1993 Development of the human brain: in vivo quantification of metabolite and water content with proton magnetic resonance spectroscopy. Magn Reson Med 30:424-437

16. Toft PB, Leth H, Lou HC, Pryds O, Henriksen O 1994 Metabolite concentrations in the developing brain estimated with proton MR spectroscopy. J Magn Reson Imaging 4:467-680

17. Slager UT, Berggren RL, Marubayashi S 1977 Non-ketotic hyperglycinemia: report of a case and review of the clinical, chemical and pathological changes. Ann Neurol $1: 399-402$

18. Hutchesson A, Preece MA, Gray G, Green A 1997 Measurement of lactate in cerebrospinal fluid investigation of inherited metabolic diseases. Clin Chem 43:158 161

19. Lu FL, Wang PJ, Hwu WL, Tsou Yau KI, Wang TR 1999 Neonatal type of hyperglycinemia. Pediatr Neurol 20:295-300

20. Byrd DJ, Krohn HP, Winkler L, Steinborn C, Hadam M, Brodehl J, Hunneman DH 1989 Neonatal pyruvate dehydrogenase deficiency with lipoate responsive lactic acidemia and hyperammonemia. Eur J Pediatr 148:543-547

21. Marliss EB, Aoki TT, Toews CJ, Felig P, Connon JJ, Kyner J, Huckabee WE, Cahill Jr GF 1972 Amino acid metabolism in lactic acidosis. Am J Med 52:474-481

22. Kowaloff EM, Phang JM, Granger AS, Downing SJ 1977 Regulation of proline oxidase activity by lactate. Proc Natl Acad Sci USA 74:5368-5371

23. Ogawa H, Gomi T, Takusagawa F, Fujioka M 1998 Structure, function and physiological role of $N$-methyltransferase. Int J Biochem Cell Biol 30:13-26

24. Wu G, Morris Jr SM 1998 Arginine metabolism: nitric oxide and beyond. Biochem J 336:1-17

25. Butterworth RF 1998 Effect of hyperammonemia on brain function. J Inherit Metab Dis 1(suppl):6-20

26. Bode BP, Souba WW 1997 Glutamine transport and human hepatocellular transformation. JPEN J Parenter Enteral Nutr 23(suppl):S33-S37

27. Curthoys NP, Watford M 1995 Regulation of glutaminase activity and glutamine metabolism. Annu Rev Nutr 15:133-159

28. Tate SS, Leu FY, Meister A 1972 Rat liver glutamine synthetase: preparation, properties and mechanism of inhibition by carbamyl phosphate. J Biol Chem 247:5312-5321

29. Cimino F, Tramonti C 1973 Mammalian glutamine synthetase: molecular and regulatory properties. Acta Vitaminol Enzymol 27:135-144

30. Dringen R, Verleysdonk S, Hamprecht B, Willker W, Leibfritz D, Brand A 1998 Metabolism of glycine in primary astroglial cells: synthesis of creatine, serine and glutathione. J Neurochem 70:835-840

31. Cohen DM 1997 Inhibition of glutamine synthetase induces critical energy threshold for neuronal survival. Ann NY Acad Sci 826:456-460

32. Hertz L, Yu AC, Kala G, Schousboe A 2000 Neuronal-astrocytic and cytosolicmitochondrial metabolite trafficking during brain activation, hyperammonemia and energy deprivation. Neurochem Int 37:83-102

33. Bachmann C, Mihatsch MJ, Baumgatner RE, Brechbühler T, Bühler UK, Olafsson A, Ohnacker H, Wick H 1971 Nicht-ketotische Hyperglyzinämie: perakuter Verlauf im Neugeborenealter. Helv Paediatr Acta 26:228-243

34. Caldwell J, Moffat JR, Smith RL 1976 Post-mortem survival of hippuric acid formation in rat and human cadaver tissue samples. Xenobiotica 6:275-280 